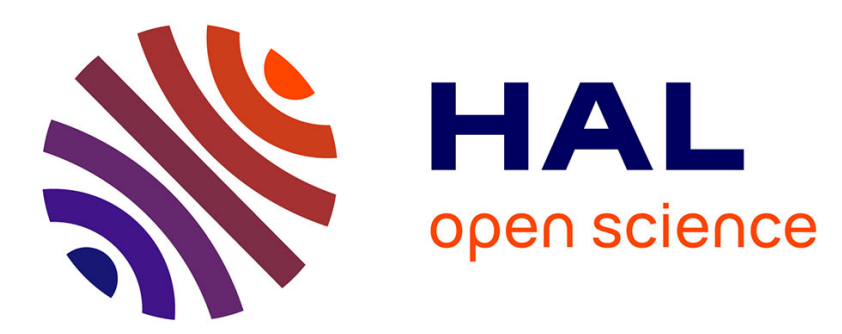

\title{
La part et le rôle joués par les modes informels de règlement des litiges dans le développement d'un pluralisme judiciaire (Etude comparative France-USA) Jean-Pierre Bonafé-Schmitt
}

\section{- To cite this version:}

Jean-Pierre Bonafé-Schmitt. La part et le rôle joués par les modes informels de règlement des litiges dans le développement d'un pluralisme judiciaire (Etude comparative France-USA). Droit et Société, 1987, 6 (1), pp.263-283. 10.3406/dreso.1987.960 . hal-01509361

\section{HAL Id: hal-01509361 \\ https://hal.science/hal-01509361}

Submitted on 18 Apr 2017

HAL is a multi-disciplinary open access archive for the deposit and dissemination of scientific research documents, whether they are published or not. The documents may come from teaching and research institutions in France or abroad, or from public or private research centers.
L'archive ouverte pluridisciplinaire HAL, est destinée au dépôt et à la diffusion de documents scientifiques de niveau recherche, publiés ou non, émanant des établissements d'enseignement et de recherche français ou étrangers, des laboratoires publics ou privés. 


\section{La part et le rôle joués par les modes informels de règlement des} litiges dans le développement d'un pluralisme judiciaire (Etude comparative France-USA)

Jean-Pierre Bonafé-Schmitt

\section{Citer ce document / Cite this document :}

Bonafé-Schmitt Jean-Pierre. La part et le rôle joués par les modes informels de règlement des litiges dans le développement d'un pluralisme judiciaire (Etude comparative France-USA). In: Droit et société, $\mathrm{n}^{\circ} 6,1987$. Normes, déréglementation, économie. pp. 263-283;

doi : $10.3406 /$ dreso.1987.960

http://www.persee.fr/doc/dreso_0769-3362_1987_num_6_1_960

Document généré le 06/06/2016 


\begin{abstract}
The increase of the number of cases dealt with in court is often presented as an increase in the judiciary character of our social relations. During the last ten years, the number of cases dealt with by the courts has increased by $50 \%$. Without denying the importance of this increase, we can ask ourselves about the real nature of this phenomenon and try to observe if this quantitative increase dots not hide another reality.

Historically, courts have never had the monopoly of the settlementt of disputes. There has always existed an "informal justice" like the disciplinary committee in companies, associations, the different kinds of private arbitrations, particularly in commercial matters.

What might appear as new is the fact that the state itself has been developing for a few years "extra judicial procedures" like "conciliators", "Boite postale 5000"... With these new procedures of settlement, the state tries to obtain more "flexibility" in the judicial System.
\end{abstract}

\title{
Résumé
}

L'augmentation croissante du contentieux soumis à la justice est le plus souvent présentée comme une juridicisation croissante des rapports sociaux ou comme une étatisation plus poussée de nos sociétés. C'est ainsi qu'au cours des dix dernières années, le nombre d'affaires traitées par les juridictions s'est accru de $50 \%$. Sans nier l'importance de cet accroissement, on peut toutefois s'interroger sur la véritable nature de ce phénomène et rechercher si cet accroissement quantitatif ne dissimule pas une autre réalité.

II faut reconnaître que, quels que soient le type de société, les périodes historiques, l'appareil judiciaire n'a jamais eu le monopole du règlement des litiges : il a toujours persisté, sous une forme plus ou moins développée, ce que l'on pourrait appeler une "justice informelle", comme les "juridictions disciplinaires" dans les entreprises, les associations, les différentes formes d'arbitrage, notamment en matière commerciale.

Ce qui peut apparaître nouveau, c'est que l'Etat lui-même développe depuis quelques années des procédures extrajudiciaires, ce que certains ont appelé des "circuits de dérivation", comme les "conciliateurs", les "modérateurs", les "Boîtes postales 5000"... Avec la mise en place de ces mécanismes plus souples de règlement des conflits, l'Etat cherchait à obtenir une plus grande "flexibilité" de l'appareil judiciaire. 


\title{
La part et le rôle joués par les modes informels de règlement des litiges dans le développement d'un pluralisme judiciaire (Etude comparative France-USA)*
}

\author{
Jean-Pierre BONAFE-SCHMITT**
}

SUMMARY

The increase of the number of cases dealt with in court is often presented as an increase in the judiciary character of our social relations. During the last ten years, the number of cases dealt with by the courts has increased by $50 \%$. Without denying the importance of this increase, we can ask ourselves about the real nature of this phenomenon and try to observe if this quantitative increase does not hide another reality.

Historically, courts have never had the monopoly of the settlement of disputes. There has always existed an informal justice" like the disciplinary committee in companies, associations, the different kinds of private arbitrations, particularly in commercial matters.

What might appear as new is the fact that the state itself has been developing for a few years "extra judicial proce. dures" like "conciliators", "Boite postalt 5000"... With these new procedures of settlement, the state tries to obtain more "flexibility" in the judicial system.

\section{RESUME}

L'augmentation croissante du contentieux soumis à la justice est le plus souvent présentée comme une juridicisation croissante des rapports sociaux ou comme une étatisation plus poussée de nos sociétés. C'est ainsi qu'au cours des dix dernières années, le nombre d'affaires traitées par les juridictions s'est accru de $50 \%$. Sans nier l'importance de cet accroissement, on peut toutefojs s'interroger sur la véritable nature de ce phénomène et rechercher si cet accroissement quantitatif ne dissimule pas une autre réalité.

Il faut reconnaitre que, quels que soient le type de société, les périodes historiques, l'appareil judiciaire n'a jamais eu le monopole du règlement des litiges: il a toujours persisté, sous une forme plus ou moins développée, ce que l'on pourrait appeler une "justice informelle", comme les "juridictions disciplinaires" dans les entreprises, les associations, les différentes formes darbitrage, notamment en matière commerciale.

Ce qui peut apparaître nouveau, c'est que l'Etat lui-même développe depuis quelques années des procédures extrajudiciaires, ce que certains ont appelé des "circuits de dérivation", comme les "conciliateurs", les "modérateurs", les "Boîtes postales 5000 "... Avec la mise en place de ces mécanismes plus souples de règlement des conflits, l'Etat cherchait à obtenir une plus grande "flexibilitê" de l'appareil judiciaire.

* Cet article reprend le contenu d'une communication

faite au Congrès annuel de l'Association Law and So- 
Durant la même période, c'est-à-dire dans les années soixante-dix, l'Etat des deux côtés de l'Atlantique s'est lancé progressivement dans une politique de déjudiciarisation et de délégalisation. Cette politique visait à répondre à un double mouvement de fond, apparemment contradictoire, qui a pris naissance au milieu des années soixante. C'est en effet à partir de cette période que s'est catalysé, d'une manière diffuse, un mouvement de critique, de défiance à l'égard du fonctionnement de l'appareil judiciaire. Le réquisitoire portait sur un certain nombre de points, tels que la lenteur, le coût, la complexité de la procédure, sans oublier l'archaisme du langage judiciaire. Mais ce mouvement de critique s'est doublé paradoxalement d'une demande de participation plus active des justiciables à l'administration de la justice, à travers ce que l'on a appelé en France "la démocratisation" de l'appareil judiciaire. Cette demande de démocratisation a été surtout le fait de mouvements organisés, comme les associations de locataires, de consommateurs, de justiciables, qui interviennent directement dans le processus de règlement des conflits à travers la défense de leurs

ciety à Chicago - 29/5 au 1/6/1986 - Il est tiré d'une recherche menée en collaboration avec D. Picon, C. Gerard, P. Porcher: "Les justices du quotidien: les modes formels et informels de règlement des petits litiges", Lyon, GLYSI/Univ. Lyon II. 1986, 304+5 p. Cette recherche a été financée par le Commissariat Général du Plan et le Ministère de l'Education. Dans le cadre de cette recherche, nous avons procédé à l'analyse de deux juridictions: le Tribunal d'Instance de Villeurbanne pour la France et le "Dane County Small Claims Court" pour les USA et de quatre institutions extra-judiciaires: la Boîte postale 5000, l'Union Fédérale des Consommateurs du Rhône, le Groupement d'Action judiciaire pour la France, le "Milwaukee Mediator Center", le "Dispute Settlement of Racine", le "Case Mediation Program" (Wisconsin) pour les USA.

** GLYSI-CNRS/Université Lyon II. adhérents.

\section{La déjudiciarisation: une ré- ponse à la crise du modèle ra- tionnel de régulation judiciai-} re.

Pour bien saisir le caractère novateur de ce mouvement de déjudiciarisation, il aurait fallu le mettre en parallèle avec le développement de notre propre histoire judiciaire. Cette comparaison ne peut être faite en raison de la quasi-inexistence dans notre pays d'une discipline équivalente à la "legal history" américaine.

Aux USA, en effet, on trouve un certain nombre de recherches qui ont pour objet d'analyser ces phénomènes de délégalisation et de déjudiciarisation sur un plan historique(1). Ces études se proposent de montrer que les politiques de délégalisation, notamment celles mises en oeuvre au cours des années 1900-1930, ne visaient qu'à corriger les effets pervers, les dysfonctionnements nés d'une application trop stricte du formalisme légal sans vraiment remettre en cause le phénomène dominant de rationalisation judiciaire.

Le mouvement contemporain de déjudiciarisation ne semble pas être de même nature. Ce ne sont plus les dysfonctionnements d'un système qui sont en cause, mais le système lui-même, fondé sur une rationalisation continue des procédures de régulation des conflits. Ayant établi ce constat de crise du "modèle rationnel", on ne peut donc faire l'économie d'un débat sur l'émergence, à partir de ce mouvement de déjudiciarisation, d'un "nouveau modèle" de régulation des litiges. Cette crise $\mathrm{du}$ "modèle rationnel" semble toucher l'ensemble 
des modes de régulation sociale, qu'ils soient centralisés comme celui en vigueur en France, ou décentralisés comme celui des USA. Sans entrer dans le détail de chaque système, on peut dire que le système français repose sur des instruments de régulation centralisés, fonctionnant sur un modèle hiérarchique, faisant appel à une conception du droit très réglementaire(2), alors que le système américain serait plus décentralisé et contractuel.

Cette conception centralisée des modes de régulation sociale provient essentiellement de notre culture colberto-jacobine, caractérisée par un interventionnisme étatique à outrance et touchant l'ensemble des secteurs de la vie sociale. Dans un tel modèle, où elle est entièrement structurée par et autour de l'Etat, la société apparait comme figée "en monolithes qui ne savent plus communiquer entre eux que par la médiation de l'Etat partout sollicitén (3). Ainsi, pour faire face à la multiplication des situations juridiques nouvelles, le législateur avait pris pour habitude de créer des juridictions spécifiques venant se surajouter aux anciennes déjà existantes: tribunaux paritaires des baux ruraux, de pensions, de dommages de guerre, commission de Sécurité sociale, Cour de Sûreté de l'Etat... Si au départ le souci dominant qui avait amené le législateur à créer ces juridictions spécialisées pouvait être celui d'accroître l'efficacité de l'appareil judiciaire, à terme cette prolifération des tribunaux a entraîné une bureaucratisation de la justice. On mesure aujourd'hui les effets pervers de cette prolifération dans la mesure où cet accroissement du nombre des organismes juridictionnels a multiplié les risques d'incertitude de compétence qui "aboutissent en définitive à accroître les lenteurs de la justice" (4).

La société américaine, en raison de la structure fédérale de l'Etat, de la multiplicité des pouvoirs, n'a pas été atteinte par le syndrome étatiste et a conservé une certaine autonomie par rapport à l'Etat, ce qui se manifeste notamment par la mise en oeuvre d'instruments de régulation non étatiques. C'est le cas précisément en matière de relations du travail où les procédures d'arbitrage prévues dans les conventions collectives s'apparentent à une véritable justice privée(5). D'une manière simplifiée, on pourrait dire que la régulation sociale en France est une régulation essentiellement étatique, fondée sur l'édiction de règles générales, impersonnelles et impératives, qui ne laissent aucune marge d'initiative aux acteurs. Ce corset réglementaire, qui enserre la société dans des règles rigides, représente un obstacle à une autre régulation des litiges par la société.

Aux USA, il existe aussi une intervention étatique en matière de régulation sociale, mais celle-ci, au lieu de fixer des règles de comportements, les droits et devoirs de chacun, cherche plutôt à fournir un cadre aux relations entre les parties, à définir des procédures permettant la régulation des conflits. Mais cette apologie du "due process" fait que ce pays souffre du "délire de procédure" qui se révèle aussi sclérosant que le "délire réglementaire" dont est atteinte la France(6). Le développement considérable du contentieux, qui fait des Etats-Unis la société la plus litigieuse du monde, a provoqué un grippage de l'appareil judiciaire dont les principales manifestations sont la longueur des délais, le coût de la procédure... Cette situation explique l'encombrement des rôles des tribunaux des grandes villes américaines. Ainsi, à Boston, Chicago, New-York, certaines procédures durent plus de quatre années(7). En France, la situation n'est pas meilleure; ainsi la durée moyenne des procédures est de l'ordre de deux années de- 
vant le Tribunal de Grande Instance de Lyon. A la longueur de ces délais, qui s'apparente à de véritables dénis de justice, il faudrait ajouter la complexité et le coût de la procédure, qui constituent autant de barrières à un réel accès à la justice. En effet, cette recherche d'une plus grande rationalisation du système judiciaire dans un souci d'efficacité s'est faite le plus souvent à partir de la mise en oeuvre de principes empruntés au management scientifique, d'où la prolifération de structures spécialisées, la complexité des règles de procédure nécessitant le plus souvent lintervention de professionnels du droit.

Ce modèle de fonctionnement de l'appareil judiciaire, avec tous ses effets induits, n'est plus adapté, on le comprend aisément, à l'évolution d'une société vers une plus grande décentralisation, une demande de participation plus active des citoyens, la recherche d'une plus grande autonomie par rapport aux structures étatiques. Ce décalage s'explique par le retard avec lequel les "gestionnaires" de l'appareil judiciaire ont découvert la théorie des relations humaines, c'està-dire la nécessité de tenir compte des comportements sociaux dans le mode de régulation des litiges.

\section{Le mouvement de déjudiciari- sation}

A la lecture des statistiques judiciaires, il semblerait que notre société devienne de plus en plus conflictuelle, mais il faudrait peut-être nuancer ce jugement car, dans le passé, une bonne partie des petits litiges était régulée au sein de la famille, du quartier et autres structures communautaires. Aujourd'hui, les phénomènes d'urbanisation, de mobilité sociale, ont remis en cause ces lieux de socialisation, de régulation des conflits et, par voie de conséquence, ont amené l'Etat à intervenir dans ces domaines particuliers de la vie sociale.

Pour faire face à cette situation, les Etats ont continué à développer leurs moyens traditionnels, c'est-à-dire à favoriser un plus grand accès à la justice, mais se sont aussi attachés à développer un certain nombre d'initiatives ayant pour objet de reconstituer des lieux de socialisation où pourraient être régulés ces conflits liés à la vie quotidienne (litiges liés aux rapports de voisinage, à la vie familiale, au domaine de la consommation...), un type de contentieux qui, de par sa nature, mais aussi pour d'autres raisons (coût, délais, distance sociale), échappait jusqu'ici aux juridictions traditionnelles.

La mise en oeuvre de ces institutions constitue, il faut le souligner, un tournant dans les politiques développées par les Etats en matière de régulation sociale, car jusqu'ici c'était du côté de l'appareil judiciaire qu'ils se tournaient dans leur recherche d'une plus grande institutionnalisation des modes de règlement des conflits. Parallèlement à ces initiatives, les Etats des deux côtés de l'Atlantique se sont lancés aussi dans des politiques de "délégalisation" de certains conflits. Les exemples les plus connus de cette politique sont les procédures de divorce par consentement mutuel, ou encore les régimes d'indemnisation sans faute en matière d'accident de la circulation(8).

Au cours de ces mêmes années soixante-dix, on a vu aussi se multiplier les formes d'aide judiciaire pour faciliter l'accès à la justice des couches sociales les plus défavorisées. Malgré leur apparent succès, ces mesures d'aide, destinées à égaliser les positions des parties dans les procédures en remédiant à la disparité économique et sociale, ne 
sont pas toujours adaptées à la résolution de certains types de conflits(9). En effet, le modèle judiciaire de résolution des conflits, qui repose sur l'opposition d'intérêts (adversary model) n'est pas adéquat dans le cas de litiges opposant des parties engagées dans des relations continues comme en matière familiale ou de voisinage.

\section{Les initiatives étatiques}

Pour répondre à ce type de situation, les Etats, surtout aux USA, se sont attachés à développer des initiatives alternatives au modèle judiciaire, privilégiant d'autres techniques de résolution des conflits, à savoir la médiation, la conciliation, l'arbitrage.

\section{Les initiatives américaines}

C'est surtout aux USA, si l'on se réfère à la situation française, que l'Etat a eu la politique la plus volontariste en matière de déjudiciarisation des modes de règlement des conflits. Cette politique visait non seulement à promouvoir les techniques de médiation, de conciliation au sein des institutions judiciaires, mais elle allait aussi, contrairement à l'évolution passée, restituer à la société civile des prérogatives en matière de résolution des conflits en donnant naissance au mouvement des "Neighborhood Justice Centers".

Le développement de ce programme de médiation, aussi bien à l'intérieur qu'à l'extérieur de l'appareil judiciaire, devait permettre la réalisation de quatre objectifs(10):

- l'amélioration de l'accès à la justice, en réduisant les délais, le coût de la procédure, en facilitant la tenue des audiences à des jours et heures convenant aux parties;
- l'amélioration de l'efficacité des instances judiciaires, le transfert d'une partie du contentieux aux "centres de médiation" devant permettre aux juridictions de traiter d'une meilleure manière les cas qui leur seraient soumis;

- l'amélioration des modes de traitement des litiges car les parties seront alors plus à même d'explorer les causes profondes qui sont à l'origine du litige, sans être tenues par les restrictions imposées par les règles de procédure, la présence des avocats comme intermédiaires obligés dans les discussions entre les parties;

- l'amélioration des relations sociales, les programmes de médiation devant, selon les auteurs de ces réformes, permettre une plus grande participation des citoyens dans la résolution des conflits, une réduction des tensions sociales et aboutir ainsi à une amélioration des relations sociales et de la qualité de la vie.

La réalisation de ce programme ambitieux donna lieu à la création de nouvelles institutions comme "l'Office for Improvements in the Administration of Justice ${ }^{n}$ (OIAJ) ou à des développements législatifs avec le "Federal Dispute Resolution Act" de 1980, qui devint la charte du mouvement des "Neighborhood Justice Centers" (NJC).

Il faut rechercher les origines de ce mouvement dans les différentes expériences qui se déroulèrent au cours des années soixante-dix. Parmi les plus célèbres, on peut retenir celle de Philadelphie avec le "Philadelphia Municipal Court Arbitration", tribunal créé en 1969, celle de Colombus (Ohio) avec le "Night Prosecutor Program", 
établi en 1971, et enfin, à.Rochester (New-York) avec le "Community Dispute Service" soutenu par 1' American Arbitration Association". Très vite, l'Etat Fédéral s'intéresse à ce type d'expériences en les soutenant financièrement par le biais du "Law Enforcement Assistance Agency" (LEAA). En 1974, le projet de Colombus fut désigné comme "Exemplary Project" par la direction du LEAA. La plublicité faite autour de ces expériences allait susciter de nouvelles initiatives, notamment à Miami ou encore à Boston.

A la différence de la France, le milieu judiciaire, c'est-à-dire les organisations de magistrats et d'avocats, s'est plutôt montré favorable à ce type d'expériences, comme en témoignent les débats tenus au cours de la "National Conference on the Cause of Popular Dissatisfaction with the Administration of Justice". Cette conférence, plus connue sous le nom de "Pound Conference", fut organisée en Avril 1976 à l'initiative de l' "American Bar Association" (ABA), de la "Judicial Conference of the United States" et de la "Conference of Chief Justices". Elle avait pour objet, comme son nom l'indique, d'analyser les problèmes rencontrés par la justice dans le traitement des litiges. Elle connut un certain nombre de suites, dont notamment la publication d'un rapport: "Report of the Pound Conference follow up Task Force", la création au sein de l'ABA d'un "Committee on the Resolution of Minor Disputes". Dans le rapport précité, les auteurs firent un certain nombre de recommandations en matière de réforme de la justice, dont celles concernant le développement de modes alternatifs de résolution des petits litiges (11).

Deux ans après la publication de ce rapport, le Département de la Justice concrétisa ces recommandations en finançant trois expériences pi- lotes de résolution extra-judiciaire des conflits: les "Neighborhood Justice Centers" d'Atlanta, de Kansas City et de Los Angeles. Cette reconnaissance officielle, avec toute la publicité faite autour de ces expériences pilotes, allait stimuler le mouvement de déjudiciarisation. Il touche aujourd'hui plus de trente Etats, et on dénombra en 1982 environ 250 "Minor Disputes Centers" (pour reprendre la dénomination utilisée par Daniel Mc Gillis) qui ont pour nom: "Citizen Dispute Settlement Center", "Community Dispute Mediation Center..." (12).

Ce mouvement de réforme souffla aussi sur l'institution judiciaire elle-même, où des expériences visant à promouvoir les techniques de conciliation, de médiation, d'arbitrage furent menées au sein des juridictions de première instance. Ainsi, l'Etat du Maine, au cours de l'année 1977, mit en place un programme de médiation dans un certain nombre de "Small Claims Courts"(13). Dans ces juridictions, une fois l'affaire inscrite, le juge propose aux parties de choisir, soit la procédure de médiation, soit celle du jugement. Si la plupart des juges soulignaient que la médiation n'était pas obligatoire, il arrivait aussi que quelques-uns renvoyaient d'office, c'est-à-dire sans rechercher l'accord des parties, des affaires entre les mains des médiateurs.

L'arbitrage, tout comme la médiation, fut à nouveau mis en valeur avec le développement des "Court-Annexed Arbitrations". Dans le cadre de ces procédures d'arbitrage, c'est le juge qui renvoie une affaire à un arbitre, un avocat ou un non professionnel, selon les projets: Un certain nombre d'Etats ont développé des programmes de "CourtAnnexed Arbitration", comme l'Ohio, New-York, la Pensylvanie, le Michigan... Pour être complet sur cette question, il nous faudrait citer aussi 
l'ensemble des programmes d'arbitrage institués en matière d'accident automobile dans le cas de défaut d'assurance ("Uninsured Motorist Arbitration"), les procédures d'arbitrage instaurées en matière de consommation par les "Better Business Bureaus" ainsi que les programmes ou procédures de ce type concernant le domaine médical(14).

\section{Les initiatives françaises}

Dans ce mouvement d'expérimentation de structures alternatives à la justice, les premières initiatives françaises, si l'on se réfère aux expériences étrangères comme celles par exemple nord-américaines, semblaient sous-tendues plutôt par des logiques administratives ou gestionnaires que par un programme mobilisateur à l'image du "Dispute Resolution Act" voté par le Congrès américain en 1980. C'est en effet dans cette perspective purement gestionnaire qu'il faut resituer la création des conciliateurs, des Boîtes Postales 5000, des modérateurs dans le secteur de l'équipement, des médiateurs médicaux à la fin des années soixantedix. C'est pour faire face à ce que l'on a appelé un "contentieux de masse" et un "engorgement" de l'appareil judiciaire que furent institués ces "circuits de dérivation".

Ce mouvement d'expérimentation se cantonne au départ au seul domaine civil. Nous nous limiterons, dans le cadre de cet article, à présenter seulement les grandes lignes des différents projets. L'institution des conciliateurs, en 1977, relève d'une initiative du Ministère de la Justice de l'époque, qui limita au départ l'expérience à quatre départements. Après cette phase expérimentale, jugée concluante, des conciliateurs furent nommés sur l'ensemble de la France. C'est ainsi que l'on comptait plus de 1000 conciliateurs en
1980. Ce sont des bénévoles nommés par les Premiers Présidents des Cours d'Appel, avec pour mission de chercher à régler à l'amiable les petits litiges entre particuliers. Ils exercent leur fonction dans le cadre du canton, comme les anciens juges de paix. Mais la mise en place des conciliateurs a entraîné un certain nombre de réactions au sein du monde judiciaire qui craignait de voir se créer une "justice parallèle". Après la victoire de la gauche en 1981, le nouveau Ministre de la Justice, apparemment très sensible à ces critiques, a adressé une circulaire aux chefs des Cours d'Appel, leur indiquant qu'il serait "souhaitable de ne pas intensifier le recrutement des conciliateurs". Sans être opposé au principe de la conciliation, le projet de la Chancellerie était de développer celle-ci dans le cadre des institutions judiciaires, notamment du Tribunal d'Instance. Un projet de décret fut présenté en 1985 au Conseil d'Etat qui le rejeta au motif que la réforme devait faire l'objet d'un texte législatif.

Les $B P 5000$ furent créées en 1976, à l'initiative du Secrétariat d'Etat à la Consommation de l'époque. Elles avaient pour mission de chercher à résoudre d'une manière amiable les litiges relatifs au droit de la consommation notamment par le moyen de commissions de conciliation tripartites (Administration de la concurrence et de la consommation, associations de consommateurs et syndicats professionnels). Dès le départ, cette initiative allait aussi rencontrer l'hostilité des magistrats qui reprochaient à ce système d'empiéter sur leurs compétences. De même, les grandes organisations de consommateurs allaient se montrer hostiles à ce type d'initiative. Malgré ces oppositions multiples, les BP 5000, avec des différences notables selon les départements, allaient connaître en moyenne un relatif succès. Ainsi, en 1981, les BP 5000 , dans leur ensemble, ont reçu 27385 lettres. 
Comme pour les conciliateurs, cette institution n'a pas échappé à la critique des nouveaux ministres de l'après-mai 1981, qui se proposaient de la remplacer par une sorte de conseil de prud'hommes de la consommation. Mais ce projet ne vit jamais le jour.

Face à cet accroissement du contentieux de masse, la gauche au pouvoir ne resta pas inactive. Elle multiplia, tant sur le plan civil que pénal, des commissions, groupes d'études, ayant pour fonction de faire des propositions en matière de réforme de la justice. On peut rappeler les commissions Pesce, Bonnemaison, pour ne citer que les plus connues, sans oublier celle du Sénateur Edgar Tailhades, qui donna lieu à la rédaction d'un rapport au titre évocateur: "La modernisation de la justice" (15). Cette volonté de réforme des modes de régulation des litiges allait se concrétiser sur le terrain par l'instauration d'un certain nombre d'initiatives, plus ou moins expérimentales selon les cas. Sans prétendre être exhaustif, il faudrait noter, dans le domaine du logement, la création des Commissions départementales des rapports locatifs (CDRL). Ces commissions ont été construites sur le modèle prud'homal, c'est. à-dire qu'elles sont composées, sur une base paritaire, de représentants d'organisations de bailleurs et de locataires. Leur rôle est de s'efforcer de trouver, notamment par le biais de la conciliation, une solution aux litiges relatifs aux logements locatifs.

Une autre expérience a retenu plus particulièrement notre attention. Il s'agit de celle du Comité de conciliation de Valence, dans la Drôme, soutenu par le Conseil Communal de Prévention de la Délinquance. Ce projet est original, car il associe des habitants d'un quartier à des magistrats pour la recherche de solutions négociées dans des affaires de voisinage. Ce type d'initiative s'apparente au modèle américain des "Neighborhood Justice Centers". L'expérience se déroule sur deux quartiers où ont été mises en place des instances de conciliation composées de quatre à huit membres. Ces conciliateurs ont été nommés par l'autorité judiciaire et sont choisis en fonction "soit de leur connaissance du quartier, soit de l'intérêt qu'ils portent aux problèmes de la vie quotidienne, notamment de ceux qui naissent de la coexistence de communautés ethniques ou de générations différentes" (16).

\section{Des logiques différentes}

A ce niveau de l'analyse, il paraît important de faire ressortir les différences de logiques qui existent entre les politiques française et américaine dans ce mouvement d'expérimentation de structures alternatives à la justice. En premier lieu, les "Minor Disputes Centers" agissent en étroite relation avec l'ensemble des institutions judiciaires ou policières. C'est ainsi que bon nombre d'affaires traitées par ces centres ont fait l'objet de renvois à ces organismes, soit par les "Small Claims Courts", soit par les services de Police. Plusieurs "Small Claims Courts", comme celle du Wisconsin, rappellent, dans un document remis à l'ensemble des parties intentant une action, qu'il existe des "Mediations Centers", qui ont pour mission de les aider dans la recherche d'une solution en dehors de toute intervention judiciaire (Model for Small Claims Handbook, 1983). En France, on ne trouve pas ce type de liaison, chaque institution fonctionnant dans le domaine qui lui a été attribué sans aucun lien avec les autres. C'est en partie pour remédier à cet état de fait que le Ministère de la Justice avait proposé la réforme du statut des conciliateurs. 
En second lieu, l'objectif premier assigné aux "Minor Disputes Centers" américains n'est pas seulement d'éviter un engorgement de l'appareil judiciaire, mais également de participer à la reconstitution de liens sociaux. La mise en oeuvre de ce mécanisme passe le plus souvent par la recherche d'une implication aussi étroite que possible des membres de la communauté visée dans le processus de régulation des conflits. Ces centres sont composés de permanents (de 1 à 12 selon l'importance de l'institution), mais aussi de nombreux volontaires, pouvant varier de 30 pour le "Cambridge Dispute Settlement Center" à 380 pour le "Community Board Program" de San Francisco(17). La plupart de ces expériences avaient pour objet de promouvoir des formules de conciliation, de médiation, associant les habitants, les membres d'une communauté, à la résolution des conflits. A la différence des expériences françaises (si l'on excepte l'expérience de Valence), la grande majorité des "Minor Disputes Centers" prend en charge l'ensemble des conflits, aussi bien ceux de nature civile que ceux relevant du domaine pénal. Sur ce dernier point, les dossiers sont souvent transmis par la Police, notamment dans le cas où il s'agit de litiges liés à des problèmes de voisinage (bruit, insultes...).

\section{Les initiatives sociales}

Ces différentes expériences de déjudiciarisation suscitent, aux USA comme en France, de sévères critiques de la part de certains auteurs, qui voient dans ces diverses initiatives des formes de contrôle social(18). Pour ceux-ci, la présence de citoyens au sein des "Minor Disputes Centers" n'empêche pas que le pouvoir réel demeure dans les mains des représentants des institutions judiciaires ou de la Police(19). Par ailleurs, ils soulignent par- ticulièrement que les "Neighborhood Justice Centers" ont pour effet de neutraliser et de fragmenter l'action collective en individualisant les conflits, en les détachant de leur contexte social.

Ces critiques à l'égard de ces expériences, même si elles ne sont pas dénuées de fondements, ne permettent pas de saisir la réalité de ce mouvement de déjudiciarisation, sa nature ambivalente. En effet, si la majorité de ces expériences ont été suscitées par l'Etat, il convient aussi d'accorder une attention particulière à des initiatives qui ne relèvent pas du "machiavélisme" étatique. C.est le cas notamment de l'ensemble de celles prises par des organisations de consommateurs, de locataires, de justiciables pour intervenir dans le processus de régulation des litiges.

L'existence de ces différentes expériences nous a amené à nous interroger sur la résurgence d'une certaine conception du "communautaire", de la solidarité primaire dont la société civile avait été dépossédée par la "sociabilité étatique"(20). La reconstitution de cette forme de "sociabilité civile" serait, selon nous, facilitée par l'évolution de nos sociétés vers des systèmes complexes, hautement différenciés qui laisseraient beaucoup plus d'autonomie aux individus et aux groupes sociaux, notamment dans le domaine qui nous intéresse, celui de la gestion des conflits.

Ce type d'expériences reposant sur une logique de réappropriation du pouvoir de gérer les conflits demeure certes très minoritaire - aussi bien en France qu'à l'étranger. Parmi les expériences étrangères, on cite le plus souvent celle du "Community Board Program" de San Francisco, qui a pour objet de promouvoir une démarche communautaire et alternative en matière de justice en associant les habitants d'un quartier à la résolution des conflits. Depuis le lancement du programme 
en 1976, les artisans de ce projet ont cherché à démontrer la capacité des "communities", non seulement à prendre en charge, mais aussi à traiter les différends avant qu'ils ne dégénèrent en conflits violents. A la différence de bon nombre de "Minor Disputes Centers", qui fonctionnent comme des sous-traitants de l'appareil judiciaire, le "Community Board" poursuit un objectif inverse: réguler les litiges avant qu'ils n'entrent dans le système judiciaire traditionnel. En France, quelques associations comme SOS Agressions-Conflits à Paris ou l'association de médiation Thémis à Lyon se sont largement inspirées des principes développés par le "Community Board" pour mettre en place leur projet de médiation.

Une autre expérience mériterait aussi d'être soulignée, c'est celle des Boutiques de Droit. Le phénomène des Boutiques s'est surtout développé dans les années 1970, dans la Région parisienne et dans quelques grandes villes comme Lyon, Nantes, Montpellier, Orléans, Avignon...L'originalité de la démarche des Boutiques de Droit repose sur cette volonté d'associer les parties dans la recherche d'une solution à leur affaire, en expérimentant de nouvelles pratiques, de nouveaux modes de résolution des conflits. Ainsi en matière de divorce, les Boutiques de Droit de Paris et Lyon ont créé des "groupes divorce" pour permettre aux divorçants de régler par eux-mêmes les effets et les conséquences de leur divorce. Partant de l'idée que la question du divorce n'est pas un problème juridique mais avant tout un problème social, les militants de la Boutique de Droit de Lyon ont proposé aux membres du "groupe divorce" de ne pas se laisser enfermer dans une logique purement judiciaire. Le processus de médiation et de négociation est apparu aux militants de cette Boutique de Droit comme la procédure permettant la plus grande implication des acteurs dans le règlement de leur propre affaire. C'est sur une base négociée que les membres du "groupe divorce" ont réorganisé leurs futurs rapports en déterminant eux-mêmes le montant des pensions alimentaires, les modalités de la garde des enfants, le partage des biens du ménage...

Et c'est à partir de cette expérience que les membres de la Boutique de Droit de Lyon, associés à deux autres associations (Mouvement d'Action Sociale et San Marco), cherchent à développer un projet de médiation sur un quartier de Lyon. Les artisans de ce projet voudraient démontrer à partir d'une approche communautaire, la capacité du mouvement associatif, non seulement à prendre en charge, mais aussi à traiter les conflits, avant qu'ils ne dégénèrent en conflits violents. A la différence de bon nombre de structures de médiation qui fonctionnent comme des sous-traitants de l'appareil judiciaire, le projet de Lyon, comme le "Community Board Program" de San Francisco, poursuit l'objectif de réguler les litiges avant qu'ils n'entrent dans le système judiciaire traditionnel.

\section{Les alternatives à la justice: un pluralisme judiciaire?}

Dans chaque société, il existe, comme nous venons de le voir, une pluralité des modes de règlement des litiges, qu'ils soient formels comme le Tribunal d'Instance ou la "Small Claims Court", ou encore informels comme la BP 5000 ou le "Milwaukee Mediation Center". L'existence de cette pluralité d'instances de règlement nous a amené à nous interroger plus généralement sur l'existence de ce que l'on pourrait appeler un "pluralisme judiciaire". Sur cette question, faut-il 
s'en tenir à une conception restrictive du pluralisme judiciaire, c'est-à-dire limiter ce phénomène au champ de l'organisation judiciaire formelle, ou bien l'étendre à l'ensemble des modes de règlement formels et informels des conflits? En effet, l'appareil judiciaire, de tout temps, n'a jamais eu le monopole du règlement des litiges; il a toujours persisté ce que nous avons appelé une "justice informelle" (instances disciplinaires dans les entreprises, arbitrage...).

Pour saisir la réalité de ce pluralisme judiciaire renforcé par le mouvement de déjudiciarisation de ces dernières années, il est nécessaire de rappeler que ces modes formels et informels de règlement des litiges ne fonctionnent pas d'une manière isolée mais qu'ils s'inscrivent dans un système de médiation dont la complexité rend difficile la compréhension.

\section{Un système de médiation sociale}

La complexité de ce système de médiation est dûe en grande partie à l'interpénétration des différents modes de règlement, car l'ensemble des institutions que nous avons étudiées, qu'elles soient de création étatique ou non, n'ont pas la même fonction et ne jouent pas le même rôle. On ne peut, en effet, mettre sur le même plan la BP 5000 et les conciliateurs, ou encore l'Union Fédérale des Consommateurs (UFC) et le Groupement d'Action Judiciaire (GAJ). Les deux dernières organisations représentent des organismes de défense des intérêts de leurs adhérents, alors que les deux premières sont avant tout des organes de médiation. De même, la simple analyse informelle de ces institutions ne nous est pas apparue comme la méthode la plus pertinente, dans la mesure où ce type d'analyse se serait révélé insuffisant pour rendre compte des connexions et des interactions existant entre ces différentes instances. En effet, le règlement d'un litige ne peut être considéré comme un simple problème individuel, mais comme le révélateur du fonctionnement d'un système de régulation sociale. C'est un fait que les rôles et fonctions joués par chaque instance ou acteur en matière de résolution des conflits s'articulent les uns aux autres, créant ainsi un véritable système social. Seule une analyse en terme de "réseau social" peut restituer toute la dynamique de la demande du justiciable, au travers des différentes instances qui le conduisent par exemple du GAJ au Tribunal d'Instance.

Pour bien saisir la réalité de ce réseau, qui échappe par définition aux instruments officiels d'enregistrement, il est nécessaire de le considérer comme une "chaine d'interaction informelle ouverte", c'est-à-dire que les différentes instances le composant ne sont pas en lien direct, et qu'elles peuvent même parfois se situer dans des relations de concurrence (21). Dans le cadre de cet article, nous n'avions pas la prétention d'étudier l'ensemble des instances de médiation, mais plutôt de mettre l'accent sur l'agencement interne de ce réseau, sur les différentes interactions. Aux USA, nous l'avons vu, les institutions judiciaires et la Police renvoient un certain nombre d'affaires auprès des "Minor Disputes Centers". C'est le cas notamment avec le "Milwaukee Mediation Center", qui reçoit la grande majorité des affaires du "District Attorney Office" $(80 \%)$ ou des services de Police (10\%).

En France, on ne retrouve pas ce type de liaison entre conciliateurs, par exemple, et Tribunal d'Instance. Ces deux institutions semblent fonctionner d'une manière alternative, le tribunal représentant dans certains cas la phase ultime de 
ce processus de recours. On serait tenté de dire la même chose de la BP 5000, dans la mesure où, si la conciliation échoue, il ne reste plus à l'usager que la possibilité de saisir dans les mêmes conditions le Tribunal d'Instance. Mais, à la différence des conciliateurs, la BP 5000 ne traite pas les dossiers, elle se contente simplement de les gérer. Son rôle se limite à la transmission des dossiers aux administrations concernées, si le litige concerne le fonctionnement de celles-ci, ou à des associations de consommateurs agréées, si le litige oppose l'usager à un commerçant, un artisan ou une entreprise.

L'UFC et le GAJ fonctionnent sur un autre modèle que celui que nous venons de décrire. Ces deux institutions sont principalement des organismes de défense. Si elles utilisent les voies de la médiation, c'est dans le but de défendre les intérêts des personnes qui les ont saisies. Dans leur action, ces deux organismes sont amenés à jouer, de ce fait, une fonction à la fois d'orientation et d'assistance dans les procédures engagées par leurs adhérents. Ces différentes organisations ne se cantonnent pas simplement à dispenser des informations juridiques, elles remplissent également des fonctions de traduction et de recours. Cette opération de traduction consiste, dans un premier temps, à identifier la demande du justiciable, puis, dans un deuxième temps, à opérer une relecture de celle-ci en termes juridiques(22). L'identification de la demande du justiciable est, en effet, une fonction essentielle des instances formant ce réseau, car les moyens mis en oeuvre ne seront pas les mêmes selon le type de problèmes posés. Il est clair que, dans les cas par exemple où il y a une infraction à la loi de 1948 sur le logement, le simple rappel des sanctions pénales prévues par les textes suffit parfois à résoudre le conflit.
L'identification de la demande étant faite, il s'agit, dans un deuxième temps, de rechercher la solution satisfaisante. La production de cette solution passe par une opération particulière, l'opération de traduction "qui transforme un énoncé problématique particulier dans le langage d'un autre énoncé particulier"(23). Cette opération de traduction ne peut être faite par le justiciable seul, dans la mesure où elle fait appel à une autre terminologie, à un autre mécanisme, ne relevant plus du fait mais du droit. Cette opération n'est pas neutre, car ce processus de traduction du fait en droit s'effectue trop souvent, selon les membres du GAJ et de l'UFC, d'une manière unilatérale par les professionnels du droit, l'élément traducteur s'appropriant la démarche en la traduisant(24). Pour eux, cette fonction de traduction constitue un enjeu important, car c'est à partir de ce processus que peut être créé un nouvel ordonnancement des rapports sociaux.

On comprend donc que les opérations de traduction déterminent l'agencement interne du réseau, car, comme nous l'avons vu, il est difficile pour un justiciable d'opérer par lui-même la traduction de sa demande en termes juridiques. Cette incapacité à mettre en oeuvre "des modes de raisonnement, des catégories, des notions" qui relèvent directement du juridique le place dans des relations de dépendance à l'égard des instances auxquelles il a recours pour obtenir une formulation complète et explicite de sa demande. Cette relation de dépendance vis-à-vis des instances, que nous venons d'étudier, explique en partie cette permanence de la "clientèle" du GAJ et de l'UFC, malgré l'accroissement du nombre de lieux de consultation juridique (Palais de Justice, Mairies, Centres Sociaux). 


\section{Une justice de "deuxième classe" ou une justice "directe" ou "commu- nautaire"}

Peut-on parler de justice de "deuxième classe" ou de justice "directe" ou encore "communautaire" lorsqu'on essaie de définir la place et le rôle joué par l'ensemble de ces organes de médiation, aux origines aussi diverses que le "Milwaukee $\mathrm{Me}$ diation Center", la BP 5000, l'UFC, ou encore le GAJ. C'est un fait que ces instances sont surtout fréquentées par les "lower classes" aux USA. La part importante d'usagers de race noire, $56 \%$ pour le "Milwaukee Mediation Center", 70\% pour le "Neighborhood Justice Center" d'Atlanta, ou encore de chômeurs (56\%) dans le cas de l'expérience de Brooklyn, vient confirmer cette idée d'une justice de "deuxième classe", d'une justice "de pauvre" (25). Dans le cadre des expériences françaises, on retrouve aussi ces "laissés pour compte" de l'appareil judicaire, qui forment la majeure partie de la "clientèle" de ces organes de médiation. En ce qui concerne le GAJ, $68 \%$ de ses usagers relèvent des catégories socio-professionnelles les plus basses, c'est-à-dire des employés aux personnes non actives. C'est une population qui ne dispose pas toujours des revenus nécessaires pour faire face aux dépenses occasionnées par une procédure. Cette idée de justice de "deuxième classe" revient aussi à travers la relation de soustraitance qui existe entre les organes de médiation et les instances judiciaires et services de Police. La grande majorité des affaires traitées par les "Minor Disputes Centers" émane de l'appareil judiciaire, qui exerce ainsi un certain contrôle sur le type d'affaires concédées aux organes de médiation.

Dans certaines circonstances, ce contrôle s'apparente à une véritable dépendance, comme dans le cas du "Milwaukee Mediation Center" qui reçoit $90 \%$ de ses affaires du "District Attorney Office" et des services de Police. Pour les "Neighborhood Justice Centers" d'Atlanta et de Kansas City, ce pourcentage s'élève respectivement à $69 \%$ et $68 \%$, mais tombe à $34 \%$ pour celui de VeniceMar-Vista(26). Ce pourcentage est même négatif dans le cas de certaines expériences, comme celle du "Community Board" de San Francisco, qui ne prennent en charge que les affaires qui leur sont soumises directement(27).

Le "Community Board" et d'autres expériences comme la "Community Association for Mediation" de Pittsburgh, ou encore les initiatives prises durant les années 1976-1980 par "l'American Friends Service Committee" pour développer un certain nombre de projets communautaires, représentent une autre facette de ce mouvement de déjudiciarisation. Ce type d'expériences, même si elles restent minoritaires, traduit l'ambivalence de ce mouvement, qui ne peut être réduit à un simple appendice de l'appareil judiciaire, à une justice de "deuxième classe" ou à une justice "de pauvre". En effet, dans de nombreux domaines comme celui de la consommation, du logement mais également de la famille, la mise en oeuvre du processus de régulation des litiges par des organisations de locataires, de consommateurs, témoigne de la vitalité de cette notion de "justice directe".

Cette activité de médiation ne peut être assimilée à une "justice de pauvre" ou de "deuxième classe", dans la mesure où les usagers de ces permanences n'appartiennent pas aux classes aux revenus les plus modestes. Le domaine de la consommation préfigure peut-être plus qu'un autre l'évolution de nos sociétés vers ce modèle qu'on a appelé une "société différenciée", avec l'existence de sous-systèmes qui génèrent leurs 
propres systèmes de régulation(28). En effet, la justice "formelle", notamment le Tribunal d'Instance, en raison de son formalisme, de sa lenteur, de son coût, de sa distance, ne peut faire face d'une manière efficace au foisonnement, à l'évolution et à la complexité des rapports sociaux. L'existence de la BP 5000, les résultats obtenus par certaines organisations de consommateurs comme l'UFC en matière de négociation d'accords contractuels témoignent de l'existence d'un sous-système social où se structurent les rapports producteurs-consommateurs.

\section{Un autre contentieux}

L'analyse comparée du contentieux traité par les différentes instances informelles (BP 5000, UFC, "Milwaukee Mediation Center"...) avec celui réglé par les structures formelles (Tribunal d'Instance, "Small Claims Court"...) illustre parfaitement cette notion de "justice plurielle".

Elle permet de constater les effets pervers des normes visant à faciliter l'accès à la justice. En effet, les tribunaux sont plutôt "colonisés" par des professionnels qui utilisent la voie judiciaire pour contraindre des particuliers à exécuter leurs obligations. Ce sont eux qui introduisent la grande majorité des affaires (par exemple, $77,3 \%$ des affaires pour la "Dane County Small Claims Court" et $63,5 \%$ pour le Tribunal d'Instance de Villeurbanne). Ces demandes sont rarement contestées par les défendeurs, transformant ainsi les juridictions de première instance en véritables agences de recouvrement des créances. En revanche, la "clientèle" des instances de médiation, que ce soit la BP 5000 ou l'UFC, est surtout composée de particuliers qui ont un litige avec un professionnel. Dans le cadre de l'UFC, les particuliers forment la quasi-totalité des demandeurs $(96,6 \%)$; pour la BP 5000 , on trouve un pourcentage similaire: $95,9 \%$.

Sur le plan méthodologique, il convient de souligner que la présentation de type statistique, quantitatif, des affaires traitées par les différentes institutions ne permet pas toujours de saisir la différence de nature entre le contentieux pris en charge par les instances informelles et celui porté devant les juridictions de première instance. D'une manière générale, le contentieux traité par ces différentes institutions porte sur des sommes relativement faibles. Pour le Tribunal d'Instance, $44,1 \%$ des affaires portent sur des litiges au montant inférieur à $3000 \mathrm{~F}$; ce pourcentage passe à $66,6 \%$ pour l'UFC et à $69,1 \%$ pour la BP 5000 . Mais cette similitude de contentieux en ce qui concerne le montant des litiges ne doit pas occulter la réalité: une différence qualitative dans la nature des affaires traitées. En effet, comme nous l'avons déjà souligné, les tribunaux sont le plus souvent utilisés par les professionnels pour contraindre leur "client" à exécuter leurs obligations. Il s'agit le plus souvent des actions en paiement, ou encore des demandes de résiliation de contrat intentées par des régies immobilières, des organismes financiers, des commerçants. En revanche, la situation s'inverse si l'on se réfère aux instances informelles comme l'UFC, la BP 5000 où l'on trouve cette fois des actions en résolution $d u$ contrat pour vice de la chose ou pour non livraison, ou encore concernant les problèmes liés à. l'exécution d'une prestation de service, sans oublier tout le contentieux relatif à la mise en cause de la responsabilité professionnelle des fabricants et des vendeurs. Cette simple énumération illustre bien la nature différente du contentieux régulé par les instances formelles et informelles et renforce cette idée d'une justice plurielle qui ne peut être limitée à la seule activité de juridictions ap- 
partenant à l'ordre judiciaire.

\section{Les techniques de médiation: du juge-médiateur au médiateur-juge}

Dans la pratique, le recours à la justice ne constitue que la phase ultime d'un processus de régulation des litiges, qui a le plus souvent débuté par des discussions directes entre les parties. C'est ainsi que, dans $80 \%$ des affaires étudiées dans le cadre du Tribunal d'Instance, les parties ont cherché à trouver, avant d'engager une action judiciaire, une solution à leur problème. Le plus souvent, ces moyens extra-judiciaires de résolution des conflits se résument à des discussions directes entre les parties $(51,9 \%)$ ou à la mobilisation d'autres moyens, comme par exemple le recours à un huissier $(38,6 \%)$. Ces démarches sont rarement couronnées de succès, même dans le cas de la recherche d'un arrangement partiel (moins de $2 \%$ des affaires).

L'échec de ces différentes tentatives extrajudiciaires de résolution des conflits explique d'une part la faiblesse du taux des conciliations devant le Tribunal d'Instance et d'autre part, la proportion importante des décisions rendues "par défaut" ou encore "réputé contradictoire".

A la simple lecture des statistiques établies par le Tribunal d'Instance de Villeurbanne, on serait tenté de conclure à l'échec de la conciliation comme mode de résolution des conflits dans la mesure où le taux des conciliations varie selon les années de 1 à $5 \%$. Toutefois, ce jugement mériterait d'être nuancé, car il faut intégrer à ces taux le pourcentage de conciliations intervenues au cours de la procédure, mais en dehors de la juridiction. Ainsi pour le Tribunal d'Instance le taux de conciliation formelle et in- formelle varie selon les années de 7 à $10 \%$. L'étude des dossiers nous a permis de constater qu'un des motifs de désistement de la part du demandeur était d'avoir trouvé un arrangement à l'amiable. Il n'est pas rare en effet que les parties utilisent l'assignation en justice comme moyen de pression pour faire aboutir des discussions, ou pour vaincre la résistance d'un adversaire de mauvaise foi.

Un des obstacles à la réalisation d'un plus grand nombre de conciliations au sein des juridictions est aussi, comme nous l'avons déjà souligné, la part importante de décisions rendues en l'absence du défendeur. Ainsi, pour le Tribunal d'Instance de Villeurbanne, la proportion de décisions rendues par défaut s'élève à $17,2 \%$ et celles réputées contradictoires à $29,2 \%$, soit un total de $46,4 \%$. Les juridictions aux USA souffrent également de ce cancer que représente le "défaut" puisque devant la "Dane County Small Claims Court", dans $61,3 \%$ des cas, le défendeur ne se présente pas à l'audience, mettant ainsi à mal les différentes tentatives de réhabilitation de la conciliation devant les "Small Claims Courts".

Si le judiciaire l'emporte sur la conciliation, c'est-à-dire que la grande majorité des affaires se termine par un jugement (en moyenne $80 \%$ ), les tribunaux sont toutefois loin de représenter un lieu de régulation essentiellement juridique des litiges. Dans bon nombre de cas, nous l'avons vu, il n'existe aucune contestation sur le fond de la demande de la part du défendeur, celui-ci se bornant simplement à demander des délais de paiement. Ce simple rôle de ratification accordé au juge, on le retrouve aussi dans les affaires où les parties sont parvenues à un arrangement amiable et demandent au juge de le consigner dans un jugement. Dans un autre type d'affaires, les parties s'adressent au juge en lui demandant d'agir 
à titre de conciliateur. C'est surtout dans des affaires impliquant des relations continues, comme en matière familiale, que l'on retrouve le plus grand nombre d'actions demandant au juge d'agir, en quelque sorte, comme un arbitre et de se fonder sur l'équité. Cet arbitrage est surtout demandé en matière de répartition des charges du ménage au moment du divorce.

Ce recours au juge comme arbitre sur la base non pas de l'équité mais du droit, on le retrouve aussi dans des affaires relatives à la détermination de la responsabilité en matière d'accident de la circulation. Dans ce type d'affaires, opposant le plus souvent des compagnies d'assurances, il y a une "sur-argumentation" juridique et il est demandé au juge d'arbitrer le débat en indiquant quel est le point de vue qui doit l'emporter. L'ensemble de ces modes de règlement souligne le caractère $a m$ bivalent du Tribunal d'Instance, lieu complexe de régulation à la fois juridique et social des litiges.

Le recours à la justice constitue, nous l'avons déjà souligné, la phase ultime d'un processus de régulation des litiges, mais un des reproches qui est fait au mode judiciaire de résolution des conflits, notamment dans le cas où les parties se trouvent dans des relations continues, c'est que le jugement met fin à une action judiciaire, mais ne supprime pas toujours les causes profondes du conflit. Dans certaines circonstances, le procès peut, comme nous l'avons vu dans les affaires de voisinage, par le biais des demandes reconventionnelles, rendre plus antagonique le conflit initial. C'est ainsi qu'un simple "accroc" dans des relations de voisinage peut se transformer, par l'entremise de procédures judiciaires, en véritable "guerre civile". Pour ce type de conflit, le mode judiciaire de résolution basé sur l'opposition d'intérêts, la détermination d'un ga- gnant et d'un perdant ne permet nullement de résoudre un différend qui est plus de nature sociale que juridique. Le problème n'est pas de savoir quelle est la loi applicable, mais quel est le problème social qui doit être résolu(29). La prise en compte de cette dimension sociale des conflits, notamment dans les litiges impliquant des individus qui se trouvent dans des relations continues, nécessite de la part des centres de médiation la mise en oeuvre de techniques différentes de celles en vigueur dans les juridictions, c'est-à-dire la conciliation, la médiation et, dans certains cas l'arbitrage. Bien que ne disposant pas d'un pouvoir de coercition, les instances informelles arrivent à obtenir des résultats non négligeables puisque le "Milwaukee Mediation Center" arrive à concilier $45,7 \%$ des affaires qui lui sont soumises; pour la BP 5000 , le taux est de $45,4 \%$ et pour l'UFC, $35,4 \%$.

La définition de ces modèles de résolution, comme idéal-types, ne doit pas faire illusion car, dans la pratique, on assiste à une véritable perversion de ces techniques. Dans certains rapports d'évaluation, comme celui de l'expérience de Brooklyn, les auteurs ont noté que des médiateurs tendaient à adopter des rôles quasi-judiciaires en donnant des conseils juridiques aux parties(30). Dans d'autres cas, la médiation s'apparente à une réelle manipulation ou pression. Ce phénomène a été particulièrement mis en relief par Felstiner et Williams, à partir de leurs observations du type de formation dispensée par le "Mediation and Conflict Resolution" de Dorchester, qui semblait promouvoir cette ambivalence(31).

Le moyen de pression le plus utilisé consiste dans la menace faite à la partie la plus récalcitrante de renvoyer l'affaire devant les juridictions, dans le cas où un accord ne pourrait 
être obtenu. Ce type de "chantage" à la sanction judiciaire est aussi un des moyens utilisés par l'UFC ou le GAJ pour amener les adversaires de leurs adhérents à trouver un compromis. Certaines organisations, comme l' "Institute for Mediation and Conflict Resolution" (IMCR), n'hésitent pas non plus à promouvoir un modèle de déroulement de la procédure proche de celui en vigueur dans les juridictions. Le modèle proposé par l'IMCR est empreint d'un certain formalisme (à l'image de celui en vigueur au sein du "Milwaukee Mediation Center"), avec distribution de la parole, tenue d'audience (caucus) et signature d'un accord. Ce type d'institution encourage les médiateurs à agir comme des professionnels, à jouer en quelque sorte le rôle de médiateur-juge. Certains auteurs n'hésitent pas à dénoncer cette "professionnalisation" rampante, qui se manifeste notamment par les recommandations faites aux futurs médiateurs par certains, comme l'"Academy of Family Mediators" et la. "Family Mediator Association", d'avoir au moins un "master degree in behavioral sciences" ou un "law degree" avant d'exercer ce type de fonction(32).

\section{Conclusion}

Pour bien saisir l'importance de ce mouvement de déjudiciarisation, il faut considérer que la question du règlement des conflits ne peut être ramenée au seul problème de la gestion de conflits individuels mais doit être envisagée sous un angle particulier, celui du fonctionnement d'un système de régulation sociale. Un système qui est en crise dans la mesure où le modèle judiciaire de résolution des conflits qui repose sur l'opposition d'intérêts (adversary model) se révèle inapproprié pour la résolution de certains types de conflits, notamment ceux qui opposent des parties engagées dans des relations continues comme en matière de voisinage ou de relations familiales. En effet, la justice traditionnelle, en raison de son formalisme, de sa lenteur, de son coût, de sa distance, connaît de plus en plus de difficultés pour prendre en compte l'évolution et la complexité des rapports sociaux.

Ayant établi ce constat de crise du "modèle rationnel" de justice, fondé comme nous l'avons déjà souligné sur une rationalisation continue des procédures de régulation des conflits, on ne peut donc faire l'économie d'un débat sur l'émergence. à partir de ce mouvement de déjudiciarisation, d'un "nouveau modèle" ou de "nouveaux modèles" de règlement des litiges. En effet les Etats, surtout aux USA, se sont attachés à développer des initiatives alternatives au modèle judiciaire, plus souples dans la forme et privilégiant d'autres techniques de résolution des conflits, à savoir la médiation, la conciliation, l'arbitrage. Ces différentes initiatives n'ont pas pour objet de répondre à des dysfonctionnements de l'appareil judiciaire, mais plutôt de rechercher une meilleure intégration sociale, de chercher à réduire les tensions sociales, de créer des solidarités à travers une plus grande participation des citoyens dans la résolution des conflits, et d'aboutir ainsi à un perfectionnement des relations sociales. Le développement de ce mouvement des "Minor Disputes Centers" allait aussi permettre à l'Etat d'étendre son "contrôle social" sur une série de groupes sociaux, de comportements qui échappaient jusqu'ici, en raison de leur nature, au contrôle de l'appareil judiciaire formel.

Ce mouvement d'expérimentation de structures alternatives à la justice allait rompre avec une cer- 
taine forme d'interventionnisme étatique qui faisait de l'Etat l'acteur principal dans toutes les formes de médiation dans les relations sociales. Jusqu'ici, la tendance profonde en matière de médiation sociale passait par le renforcement du rôle de l'Etat, que ce soit à travers l'appareil judiciaire, ou celui de l'administration. En favorisant ces expériences de médiation "directe", l'Etat cherche, non sans difficultés, à impulser une dynamique sociale visant à faire des citoyens des acteurs à part entière en matière de résolution des conflits. Dans le cadre de cette problématique, la médiation s'apparente plus à un moyen d'action visant à la recomposition de liens sociaux qu'à une simple technique de gestion des conflits.

Mais, parallèlement à ces initiatives étatiques, se sont aussi développées un certain nombre d'expériences, de type communautaire, issues du ce mouvement associatif, représentant une autre facette de ce mouvement de déjudiciarisation. Ce type d'expériences, même si elles restent minoritaires, traduit l'ambivalence de ce mouvement, qui ne peut pas être réduit à un simple appendice de l'appareil judiciaire. Avec plus ou moins de succès ces différentes expériences, comme celles du "Community Board" de San Francisco, de SOS Agressions Conflits de Paris, tentent d'élaborer un modèle alternatif de justice.

Ce modèle repose sur l'idée que la diversité et la complexité de la vie sociale encouragent le développement de modes décentralisés de règlement des litiges, notamment dans le cadre d'entités sociales restreintes. Selon les artisans de ces projets communautaires de Justice, l'exercice direct de ces responsabilités devrait renforcer la vitalité, la stabilité des relations de yoisinage et contribuer ainsi à la reconstitution de lieux de socialisation.
L'existence de ces modes extra-judiciaires de règlement des litiges nous amène à nous interroger sur l'évolution de nos sociétés vers ce que l'on a appelé une "société différenciée", avec l'existence de sous-systèmes qui générent leurs propres systèmes de régulation(33). On ne peut pas, en effet, se contenter d'analyser le développement de ces instances de médiation comme le simple résultat d'un mauvais fonctionnement de l'appareil judiciaire; au contraire, ce processus de déjudiciarisation doit être rattaché à un mouvement plus profond, lié à l'évolution des systèmes de régulation sociale vers ce que nous avons appelé un "pluralisme judicaire", pour ne pas dire une "justice plurielle".

Pour saisir la réalité de ce pluralisme renforcé par le mouvement de déjudiciarisation de ces dernières années, il est nécessaire de rappeler que ces modes informels de règlement des litiges ne fonctionnent pas d'une manière isolée, mais qu'ils s'inscrivent dans un système de régulation juridique plus vaste, dont la complexité rend difficile la compréhension. Cette complexité est due en grande partie à l'interpénétration des différents modes et techniques de règlement, sous l'impulsion notamment des acteurs qui, en fonction de leurs objectifs ou de leurs moyens, choisissent tel ou tel mode de règlement des litiges. Les normes et les institutions juridiques constituent un des domaines où se cristallise la stratégie des acteurs, d'où la nécessité de développer une sociologie judiciaire qui ne se limite pas à la seule étude des instances judiciaires ou des règles de procédure, mais qui appréhende le règlement des litiges comme un véritable système social. 


\section{Notes:}

1. C. Harrington, "Delegalisation Reform movements: a Historical Analysis", in The Politics of Informal Justice, Academic Press Inc., 1982, pp. 35-71; et plus généralement, M. Galanter, "Reading the Landscape of Disputes: what we know (and think we know) about our Allegedly Contentious and Litigious Society", UCLA Law Review, V. $31, N^{\prime \prime} 1 / 1983$.

2. M. Crozier, Le mal américain, Fayard, 1980, pp. 295-345.

3. L. Cohen-Tanugi, "Le droit sans l'Etat" in Sur la démocratie en France et en Amérique, PUF, 1985, p. 206.

4. R. Charles, La justice en France, PUF, p. 30.

5. J.P. Bonafé-Schmitt, La création des règles dans les entreprises. Etude comparative France-USA. GLYSI, 1984, p. 180.

6. M. Crozier, Le mal américain, op. cit. pp. 295-345.

7. Policy Briefs, US Department of Justice, May 1980, p. 1.

8. Sur cette question, $\mathrm{cf} E$. Johnson, V. Kantor, E. Schwartz, "Outside the Court: a Survey of Diversion Alternatives in Civil Cases", National Center for State Courts, 1977, p. 11.

9. V. Denti, "Problèmes de la "Legal Aid" dans le monde occidental", Déviance et Société, Vol. 4, 1980, pp. 379-387.
10. Policy Briefs, Neighborhood Justice Centers, op. cit. p. 1. Sur cette question, of Mc Gillis, "Minor Dispute Processing: a Review of Recent Developments", in H. Tomasic, M. Fecky, Neighborhood Justice, Longman Inc., 1983.

11. Policy Briefs, Neighborhood Justice Centers, op. cit. p. 1.

12. Mc Gillis, "Minor Dispute Processing", op. cit. p. 64.

13. Pour un plus long développement sur cette question, cf. "Outside the Court", op. cit. p. 50 et suivantes.

14. Ibidem.

15. E. Tailhades, La modernisation de la Justice, Rapport au Premier Ministre, La Documentation Française, 1985, p. 259.

16. "Comité de conciliation? Une réponse au quotidien. L'expérience de violence", Justice $\mathrm{n}^{\prime \prime} 105 / 1985$, p. 36 .

17. Citizens Forum on the Courts, 1984.

18. R. Abel, "Règlement formel et informel des conflits. Analyse d'une alternative", Sociologie du Travail, $\mathrm{n}^{\circ} 1 / 81$.

19. R. Hofichter, "Neighborhood Justice and the Social Control Problem of American Capitalism: a Perspective", in R. Abel, The Politics of Informal Justice, vol. 1, 1982.

20. A. Melucci, "Nouveaux mouvements sociaux et action collective", Revue Internationale d'Action Communautaire, 1983, $\mathrm{N}^{\circ} 10$, p.50. 
21. J. Katuszewski, R. Ogier, Réseau total et fragments de réseaux, CERSM, Université de Provence.

22. M. Callon, L'opération de traduction comme relation symbolique. Incidence des rapports sociaux sur le développement scientifique et technique, MSH, 1975.

23. Ibidem.

24. Ibidem.

25. R. Tomasic, Mediation as an Alternative to Adjudication Rhetoric and Reality in the Neighborhood Justice Movement, Working Papers, Disputes Processing Research Program, 1980, ,2.

26. R. Cook, J. Roell, D. Sheppard, Neighborhood Justice Centers. Field Text. Executive Summary, Final Evaluation Report, February 1980, US Department of Justice.
27. "Community Boards of San Francisco, 1983", Annual Report and Comprehensive Organizational Description, San Francisco, p. 35.

28. G. Teubner, "Substantive and Reflexive Elements in Modern Law", Law and Society Review, V. 17, N"2/1983, p. 272.

29. D. Smith, "A Warmer Way of Disputing: Mediation and Conciliation", American Journal of Comparative Law, 26, 1978, p. 205.

30. R. Tomasic, Mediation as an Alternative to Adjudication, op. cit. p. 18.

31. R. Tomasic, op. cit. p. 21.

32. P. Wahrhaftig, "Non Professionnal Conflict Resolution", Villanover Law Review, vol 29, $\mathrm{n}^{\prime \prime} 6$, Novembre 1984.

33. G. Teubner, "Substantive and Reflexive Elements in Modern Law", op. cit. 


\section{L'AUTEUR}

\section{Jean-Pierre BONAFE-SCHMITT}

Né le 14/09/49. Après des études de licence et de maitrise en droit, il a obtenu, en 1980, un doctorat de 3ème cycle à l'Université Lyon III. Depuis 1978, il est chercheur au Groupe Lyonnais de Sociologie Industrielle - UA $894 \mathrm{du}$ CNRS - de l'Université Lyon II.

Ses domaines de recherche portent sur les modes formels et informels de règlement des litiges, que ce soit dans le domaine du travail (Le recours ouvrier, 1981) ou encore dans le hors travail (Les justices du quotidien, 1986), sur les systèmes normatifs dans une perspective comparative avec les USA (La création des règles dans les entreprises). Il a publié sur ces thèmes une série d'articles dans des revues spécialisées: "L'action des délégués du personnel en matière de réclamations individuelles", Droit Social, $\mathrm{n}$ " 9-10:1981; "La pratique de la négociation collective dans l'entreprise", Revue française des Affaires sociales, octobredécembre 1982; "Les nouveaux prud'hommes: un nouvel enjeu syndical", Travail et Emploi, n" 18/1983; "La médiation en France", Le Bulletin, n" 16/1986.
POUR EN SAVOIR

PLUS...

- J. FREUND, Sociologie des confits, Paris, 1983, PUF.

- R. ABEL, The Politics of Informal Justice (2 vol.), New York, Academic Press.

- M. CAPPELLETTI, Accès à la justice et Etat providence, Paris, Economica, 1984.

- L. COHEN TANUGI, Le droit sans l'Etat, Paris, PUF, 1985.

- G. GURVITCH, L'expérience juridique et la philosophie pluraliste du droit, Pedone, 1935. 